\title{
Actitudes de los Estudiantes de Pedagogía de las Regiones del Bío Bío y la Araucanía de Chile hacia la Presencia de Niños Inmigrantes en la Escuela: Análisis Diferenciales
}

\section{Attitudes of Student Teachers in the Bío Bío and Araucanía Regions of Chile towards the Presence of Immigrant Children in Schools: Differential Analysis}

\author{
Leandro Navas y Alejandra Sánchez \\ Universidad de Alicante
}

\begin{abstract}
El objetivo principal del estudio fue analizar las actitudes hacia la educación multicultural de un grupo de estudiantes chilenos de pedagogía. El diseño fue exploratorio, descriptivo y correlacional. Participaron 117 individuos de 17 a 33 años, a los que se les administró la Escala de Actitudes hacia la Educación Multicultural (Cabrera, Espín, Marín \& Rodríguez, 1997a). Los resultados indicaron que el instrumento presentó propiedades psicométricas aceptables y que las actitudes de los individuos mostraron una tendencia favorable, excepto en la variable multiculturalidad negativa. No obstante, un número importante de participantes mostró una actitud intermedia, la cual podría indicar un prejuicio sutil. Se observaron mejores actitudes en las mujeres y en los individuos que se han relacionado con personas inmigrantes.
\end{abstract}

Palabras claves: actitud, inmigración, educación multicultural, prejuicio.

\begin{abstract}
The principal objective of the study was to analyze attitudes towards multicultural education in a group of Chileans student teachers. The design was exploratory, descriptive and correlational. Onehundred and seventeen individuals aged 17 to 33 participated and were given the Multicultural Education Scale of Attitudes (Cabrera, Espín, Marín \& Rodríguez, 1997a). Results indicated that the selected instrument presented acceptable psychometric properties and the individuals' attitudes showed a favorable trend, with the exception of the variable named negative multiculturality. However, a significant number of individuals showed intermediate attitudes, which could indicate subtle prejudice. Better attitudes were observed among women and in individuals who have been associated with immigrants.
\end{abstract}

Keywords: attitude, immigration, multicultural education, prejudice.

\section{Introducción}

Por inmigrantes se entiende a la minoría étnica cuyos miembros tienen afinidades raciales, culturales y lingüísticas y que proviene del extranjero. En Chile, el censo de población de 2002 reveló que la inmigración aumentó en un 75\% durante la década 1992-2002 (Chile, Instituto Nacional de Estadísticas, 2003). Este fenómeno sigue aumentando y, actualmente, alrededor del
2\% de la población chilena está constituida por extranjeros. La Política Nacional Migratoria reconoce a Chile como receptor de cuotas significativas de inmigrantes (Chile, Presidencia de la República, 2008), procedentes, principalmente, de países como Perú, Argentina, Ecuador y Bolivia. El censo de 2002 mostró, además, que 34.006 niños extranjeros menores de 14 años residen en Chile. De estos, el 73,9\% corresponde a niños sudamericanos. Así, es razonable pen-

Leandro Navas Martínez, Departamento de Psicología Evolutiva y Didáctica, Facultad de Educación, Universidad de Alicante, España.

Alejandra Sánchez Bravo, Departamento de Psicología Evolutiva y Didáctica, Facultad de Educación, Universidad de Alicante, España.

La correspondencia relativa a este artículo debe ser dirigida a Leandro Navas Martínez, Departamento de Psicología Evolutiva y Didáctica, Universidad de Alicante, Apartado de Correos 99, 03080 Alicante, España. E-mail: leandro. navas@ua.es 
sar que este país avanza hacia una sociedad pluricultural, hecho que obliga a los científicos sociales y a los docentes a reflexionar sobre las relaciones entre las minorías que llegan del extranjero y la sociedad de acogida y sobre los efectos que la multiculturalidad puede generar en las aulas.

La inmigración y la multiculturalidad en la escuela son fenómenos recientes en la sociedad chilena que no están suficientemente considerados en la legislación actual, por lo que, para algunos, los inmigrantes constituyen uno de los grupos minoritarios cuyos derechos fundamentales (salud, trabajo y educación) se encuentran en elevados niveles de vulnerabilidad (Quesille, 2007). Por ejemplo, únicamente desde 2005 está regulado el ingreso de niños inmigrantes al sistema educativo y el acceso a este se ve dificultado, principalmente, por el desconocimiento que los administradores tienen de la legislación vigente. Lo que parece ser un trámite sencillo es engorroso y exige muchos documentos para conseguir una matrícula (Quesille, 2007).

Los países que se inician en la inmigración, como Chile, deben tener presente que los resultados que consistentemente se repiten en las escuelas con alumnos de otras culturas son los bajos rendimientos que presentan estos estudiantes (Glick \& Hohmann-Marriott, 2007; Glick \& White, 2003). En las décadas de 1960 y 1970 algunos teóricos explicaban esta situación mediante el modelo del déficit cultural: la cultura familiar de los estudiantes era "inferior" porque no los preparaba para adaptarse a la escuela. Actualmente, se rechaza esta idea al considerar que no existen culturas deficientes, sino incompatibilidades entre la cultura familiar del estudiante y las expectativas de la escuela (Gallimore \& Goldenberg, 2001). Entre las explicaciones más recientes sobre el bajo rendimiento académico de los estudiantes inmigrantes, resaltan las bajas expectativas, los prejuicios, los estereotipos de los demás (maestros y estudiantes) y las políticas de los centros educacionales (Woolfolk, 2006).

En relación con los profesores, se ha comprobado que estos recurren a diversas fuentes de expectativas, como las puntuaciones en los tests, el sexo, las calificaciones anteriores, el nivel socioeconómico, la raza y el origen cultural de sus alumnos (Archer, 1981; Navas, Sampascual \& Castejón, 1991; Van Matre, Valentine \& Cooper, 2000) y a la percepción que tienen sobre su propia capacidad para enseñar (Díaz, 2002; Jackson, 1968). De ahí se deriva el interés por conocer las actitudes de los futuros docentes ante los estudiantes inmigrantes. Quizás la forma más adecuada y sólida de aumentar las expectativas de los profesores hacia los alumnos extranjeros es modificar sus actitudes durante el proceso de formación inicial.

Uno de los problemas causados por los estereotipos culturales sobre los alumnos pertenecientes a minorías étnicas e inmigrantes es la llamada amenaza del estereotipo, que consiste en que los estudiantes estereotipados soportan una carga emocional y cognoscitiva que interfiere en sus interacciones sociales y en sus tareas mentales, lo cual podría generarles ansiedad ante las pruebas y menoscabar su desempeño (Aronson, Lustina, Good, Keough, Steele \& Brown, 1999; Steele, 1997). Además, a largo plazo, los estudiantes desarrollan estrategias de autodefensa para proteger su autoestima académica, poniendo en marcha conductas de aislamiento y de apatía (Aronson, Fried $\&$ Good, 2002).

Otro problema a considerar son los patrones evolutivos de la identidad cultural o étnica, pues, después de la familia, es en la escuela donde se adquieren los referentes culturales y se desarrolla la identidad social. La construcción de la identidad cultural comienza a temprana edad (Barron, 2007) y la adolescencia es un período especialmente importante en el establecimiento de una identidad funcional. La actitud de los maestros repercutirá significativamente en la construcción de esta identidad y en la capacidad que tengan para resolver las situaciones conflictivas derivadas de las diferencias culturales que se presenten en el aula. Díaz (2002) señala que cuando las relaciones con profesores y compañeros están basadas en el respeto mutuo, representan 
importantes fuentes de apoyo y motivación para el aprendizaje y, cuando no, se convierten en una fuente de estrés e inhiben el aprendizaje. Así, la actitud del maestro hacia estudiantes de otras culturas facilita o entorpece el proceso de identificación social, puesto que las creencias que tiene el maestro sobre los estudiantes extranjeros influyen en la forma como construyen y expresan sus identidades culturales (Brunsma, 2006; Roth, 2005).

Los prejuicios y los estereotipos que acompañan a los profesores influyen o dificultan la integración escolar de los alumnos inmigrantes. En un estudio realizado en Madrid sobre las condiciones de la integración escolar de alumnos de distintas minorías étnicas (Díaz, Baraja \& Royo, 1996) se concluye que los alumnos perciben con precisión el estilo que cada profesor muestra hacia la diversidad cultural. Así, el 10\% de los alumnos describe a sus profesores como comprometidos en la lucha contra el racismo y la discriminación y en adaptar eficazmente la educación a la diversidad; el 78\% hace referencia a un trato igualitario de los profesores hacia los españoles y los extranjeros, es decir, no hace diferencias entre estos dos grupos ni intenta adaptar la educación a sus necesidades; y el 12\% describe a los profesores discriminando negativamente a los extranjeros. Díaz et al. (1996) concluyen que el estilo de atención a la diversidad de la inmensa mayoría de los profesores se caracteriza por la pasividad, dejando a los alumnos el control de las interacciones y no intentando compensar la desigualdad de oportunidades que imponen las propias diferencias culturales de los alumnos, pero tampoco fomentándolas. Díaz (2002) señala que actuar como si no existiera la diversidad cultural suele observarse en los profesores cuando manifiestan que todos los alumnos son iguales y, por eso, no hacen diferencias entre ellos, lo que expresa el deseo de los maestros de no ser considerados discriminadores, pero también evidencia su falta de recursos para adaptarse a la multiculturalidad de los estudiantes (Jordán, 1994).

En cuanto a las variables que introducen diferencias en las actitudes hacia la inmigración, se mencionan el sexo, la edad, el nivel educativo y la competencia profesional (Molero, Navas \& Morales, 2001; Pantoja, 2006), así como la experiencia de contacto con inmigrantes (Brown, Eller, Leeds \& Stace, 2007; Brown \& Hewstone, 2005; Pettigrew \& Tropp, 2000). También se ha hallado que las actitudes de los profesores en formación hacia la multiculturalidad en la escuela son más favorables en las mujeres y en quienes se han relacionado con inmigrantes (Fernández, Aguilar \& Gutiérrez, 2002; León del Barco, Mira \& Gómez, 2007).

El rol del profesor es de trascendental importancia, porque de alguna manera sus pensamientos y actitudes hacia la multiculturalidad se plasman en su actividad docente y en sus expectativas sobre los alumnos (Ridao, 2006). La percepción, el concepto y la actitud que el docente tiene hacia el alumnado inmigrante serán elementos determinantes en su proceso educativo, en su éxito académico, en el desarrollo de su identidad social y en su integración. Además, las reivindicaciones derivadas de los derechos humanos hacen que los sistemas educativos asuman principios de igualdad de oportunidades, equidad y justicia. Sin embargo, la diversidad cultural es percibida con frecuencia como un problema más que como una ocasión para aprender sobre la variedad de los seres humanos. Lo que cabe preguntarse es cómo perciben la educación multicultural quienes próximamente tendrán que desarrollarla, es decir, los profesores en formación.

El sistema de educación superior chileno parece permanecer ajeno a estas demandas de formación, puesto que no se ha considerado necesario incluir explícitamente en los programas de formación de maestros una educación sistemática que los capacite para atender a estudiantes diferentes, de culturas diversas y aproximarse con menos prejuicios a ese alumnado. Por tanto, la valoración de las actitudes de los profesores antes de que ejerzan permite poner en marcha programas para el cambio actitudinal y para la sensibilización ante la educación multicultural. 
Teniendo en cuenta estos antecedentes, tres fueron los objetivos de esta investigación. En primer lugar, analizar las propiedades del instrumento de evaluación de las actitudes hacia la educación multicultural en el contexto universitario chileno; en segundo lugar, valorar el nivel de prejuicio manifiesto de los estudiantes chilenos hacia la educación multicultural y, por último, analizar si el sexo o la experiencia de relación con personas de otras culturas introduce diferencias en las actitudes de los futuros maestros.

\section{Método}

El diseño fue exploratorio, descriptivo y correlacional.

\section{Participantes}

Participaron 117 estudiantes (77 mujeres y 40 hombres) de la Facultad de Educación y Humanidades de tres universidades (dos estatales y una privada; todas laicas), de las regiones del Bío Bío y la Araucanía. El muestreo fue incidental, pues se trató de universidades con las que se mantienen vínculos de docencia o de investigación y existe disponibilidad de los individuos para responder al cuestionario. La edad osciló entre los 17 y 33 años $(M=20,2 ; D T=2,8)$. El 12,9\% cursaba Pedagogía Parvularia, el 37,8\%, Pedagogía General Básica y el 49,3\%, Pedagogía Diferencial. El 36,8\% de los alumnos cursaba primer año de la carrera, el 29,9\%, segundo y el 33,3\% estaba en tercer año. El 37,6\% manifestó no mantener relaciones con inmigrantes, el 3,4\% indicó que comparte barrio con ellos, el $17,9 \%$ afirmó que comparte estudio o trabajo, el 16,2\% compartía actividades sociales y el $24,8 \%$ mantenía relaciones personales (e.g., amistad, noviazgo). Estas relaciones se mantienen con personas sudamericanas y europeas.

\section{Instrumento y Variables}

Se usó la Escala de Actitudes hacia la Educación Multicultural de Cabrera, Espín,
Marín y Rodríguez (1997a) de la Universidad de Barcelona. Fue construida especialmente para valorar las creencias de los profesores acerca de la multiculturalidad en la escuela, entendida esta como la presencia en la misma de estudiantes inmigrantes. Tiene 24 enunciados ante los que se ha de manifestar el grado de acuerdo en una escala Likert de 5 puntos graduada desde Totalmente de acuerdo (4) a Totalmente en desacuerdo (0). Los ítems 3, 5, 6, 8, 10, 12, 14, 16, 18, 20, 22 y 23 están redactados en sentido negativo y en ellos se invierte la escala de puntuación.

En el estudio de Cabrera et al. (1997b), con 254 maestros y maestras españoles de educación primaria, la escala presentó una consistencia interna de $\alpha=0,88$. Está compuesta por cuatro dimensiones, determinadas a partir de una revisión de trabajos empíricos y teóricos sobre el tema, que son:

1. Efectos sobre el alumnado: se refiere a las consecuencias que produce la educación multicultural en los alumnos y expresa ideas como "la dinámica de una clase con niños de diferentes etnias favorece su socialización" o "el contacto con otras etnias que tienen los niños en situaciones educativas multiculturales les prepara mejor para adaptarse a los cambios y el futuro".

2. Efectos sobre el profesorado: se refiere a los resultados que produce la educación multicultural en los profesores, en su trabajo o en la relación con sus alumnos. Incluye ideas como "las clases con niños de diferentes minorías étnicas favorecen la innovación educativa y estimulan al profesor en la investigación de nuevas metodologías" o "la presencia de alumnos de diferentes minorías étnicas en el aula provoca un mayor estrés en el profesor".

3. Efectos sobre el trabajo en el aula: hace referencia a las implicaciones de la educación multicultural en el aula e incluye ideas como "en clases con niños de diferentes culturas se trabajan contenidos más variados y atractivos" o "la presencia de niños de diferentes culturas origina un mayor número de 
problemas de disciplina en el aula".

4. Papel de la escuela: se refiere al modo de gestionar la diversidad cultural en la escuela y presenta consideraciones como "los alumnos se interesan más por conocer otras culturas cuando en la escuela se trabaja con currículos multiculturales" o "dado que todas las culturas son tan válidas y significativas como la propia, la escuela debería acoger y asumir la diversidad cultural".

Se consideraron como variables de contraste en el análisis el sexo y el tipo de relación mantenida con un inmigrante residente en Chile (debían marcar: ninguna, compartimos barrio, estudiamos o trabajamos en el mismo lugar, compartimos alguna actividad social, tengo una relación personal) y, si la respuesta anterior era distinta a ninguna, debían indicar la nacionalidad de los inmigrantes con quienes se habían relacionado.

\section{Procedimiento}

El instrumento fue aplicado por un encuestador, previamente capacitado, el que informó a los estudiantes sobre los objetivos y características generales del estudio, les ofreció información sobre los investigadores, así como un correo electrónico para realizar consultas relativas al estudio y les solicitó su colaboración, destacando que la participación era voluntaria, confidencial y anónima. Tras obtener su consentimiento verbal, siguiendo los criterios relativos al "consentimiento informado" de la Comisión Nacional de Investigación Científica y Tecnológica, en una sesión en su aula habitual los participantes tuvieron un tiempo indefinido para completar el cuestionario, contaron con la asistencia del encuestador para aclarar dudas y no recibieron retribución.

Los participantes recibieron instrucciones impresas que indicaban: "Al leer encontrará términos como etnias o minorías étnicas. Para efectos de este estudio se refieren a grupos humanos y culturales de origen extranjero (no autóctonos)".

\section{Análisis de los Datos}

Como el instrumento de evaluación fue construido en un contexto diferente (Cataluña) al de aplicación (regiones del Bío Bío y la Araucanía de Chile), las respuestas a la Escala de Actitudes hacia la Educación Multicultural (Cabrera et al., 1997a) fueron sometidas a análisis descriptivos, de consistencia interna, por medio del coeficiente alfa de Cronbach, y de discriminación de los ítems, por medio del coeficiente de correlación $r$ de Pearson corregido entre la puntuación del ítem y la puntuación total de la escala. Considerando las respuestas a la escala como variables directamente observadas, se llevó a cabo un análisis factorial exploratorio (AFE) con el método de componentes principales (rotación Varimax con Kaiser). Con el fin de confirmar los resultados del AFE a través de un modelo de medición, se llevó a cabo un análisis factorial confirmatorio (AFC), a partir de la matriz de correlaciones policóricas entre los ítems de la escala. Se empleó el método de estimación de Mínimos Cuadrados no Ponderados (ULS), porque es más flexible en cuanto a los supuestos de los datos y al tamaño de la muestra (Jöreskog, 2001). La finalidad del modelo de medición es describir hasta qué punto las variables directamente observadas sirven para medir las variables latentes. Para realizar los análisis diferenciales, se tomaron como variables independientes el sexo y el tipo de relación con personas inmigrantes, mientras que las variables latentes se consideraron variables dependientes. Para el análisis según sexo se realizó la prueba $t$ de Student para muestras independientes. Para el análisis según el tipo de relación se realizó la prueba $U$ de Mann-Whitney para dos muestras independientes, dado que el tamaño de los grupos según el tipo de relación $(n<30)$ no aconsejaba el uso de pruebas paramétricas. El tipo de relación se dicotomizó en no tener relación o tener cualquier tipo de relación (compartir barrio, estudiar o trabajar en el mismo lugar, compartir alguna actividad social o personal -amistad o noviazgo-). 
Resultados

\section{Análisis de la Escala}

Los resultados de los análisis descriptivos se resumen en la Tabla 1, mostrando que los ítems se ajustan razonablemente a la distribución normal y presentan una adecuada capacidad de discriminación, excepto el ítem 15, el cual fue eliminado.

El AFE convergió tras 14 iteraciones. La medida de adecuación muestral fue aceptable $(\mathrm{KMO}=0,864)$ y la prueba de esfericidad de Bartlett resultó estadísticamente significativa: $\chi^{2}(276, N=117)=1223, p=0,000$. Ambos valores sugirieron la pertinencia de este análisis.

Resultaron cinco factores que, en total, explican el 59,5\% de la varianza de las actitudes hacia la educación multicultural. En la Tabla 2 se ofrece la matriz factorial rotada, de la cual se han eliminado las saturaciones inferiores a 0,30 para facilitar su lectura. Los factores obtenidos no son totalmente independientes y varios ítems saturan en más de un factor. Se siguió el criterio de mantener el ítem en el factor en el que presentaba la mayor saturación o, como en el caso del ítem 9, en el que la saturación era semejante, se incorporó al factor en el que aportaba mayor consistencia interna.

En el primer factor saturan los ítems $3,5,6,8,10,12,14,16$ y 22 y se refiere a efectos negativos de la multiculturalidad en la labor del profesor y en el rendimiento académico de los alumnos. Se ha denominado Multiculturalidad negativa, explica el $32,1 \%$ de la varianza y el coeficiente $\alpha$ es 0,83 .

El segundo factor se ha denominado Multiculturalidad positiva, explica el 11,9\% de la varianza y $\alpha$ es 0,82 . Saturan en él los ítems 11, 13, 17, 21 y 24 y hace referencia a efectos positivos de la multiculturalidad en la escuela.

El tercer factor se refiere a las respuestas de la escuela frente a la multiculturalidad y agrupa a los ítems 18, 19, 20 y 23. Por su similitud con el factor 4 de Cabrera et al. (1997b), se denominó Papel de la escuela. Explica el 5,9\% de la varianza y $\alpha$ es 0,78 .

El cuarto factor agrupa a los ítems 2, 7 y 9 y se refiere a los efectos que la educación multicultural produce en el trabajo de aula. Por su semejanza con el factor 3 de la escala original, se ha denominado Efectos en el trabajo del aula. Explica el 5,1\% de la varianza de la escala y $\alpha$ es 0,75 .

Tabla 1

Análisis Descriptivos de los Ítems de la Escala de Actitudes hacia la Educación Multicultural

\begin{tabular}{lcrrrrrr}
\hline Ítem & $\mathrm{A}$ & $\mathrm{C}$ & $r_{\text {i-total }}$ & Ítem & $\mathrm{A}$ & $\mathrm{C}$ & $r_{\text {i-total }}$ \\
\hline 1 & $-1,78$ & 3,14 & 0,45 & 13 & $-1,13$ & 0,48 & 0,57 \\
2 & $-1,64$ & 2,99 & 0,39 & 14 & $-0,29$ & $-0,60$ & 0,58 \\
3 & $-0,32$ & $-0,67$ & 0,41 & 15 & 0,53 & 1,95 & 0,19 \\
4 & $-1,50$ & 1,73 & 0,42 & 16 & $-0,38$ & $-0,15$ & 0,51 \\
5 & $-1,11$ & 0,40 & 0,43 & 17 & $-1,42$ & 1,56 & 0,58 \\
6 & 0,33 & $-0,50$ & 0,30 & 18 & $-0,61$ & $-0,72$ & 0,71 \\
7 & $-0,93$ & 0,29 & 0,55 & 19 & $-1,80$ & 3,09 & 0,60 \\
8 & $-0,48$ & $-0,77$ & 0,53 & 20 & $-1,26$ & 0,69 & 0,56 \\
9 & $-1,68$ & 2,40 & 0,61 & 21 & $-1,95$ & 3,99 & 0,55 \\
10 & $-0,57$ & $-0,35$ & 0,59 & 22 & $-0,22$ & $-0,34$ & 0,60 \\
11 & $-0,95$ & 0,25 & 0,41 & 23 & $-0,55$ & $-0,28$ & 0,55 \\
12 & $-0,55$ & $-0,10$ & 0,34 & 24 & $-0,75$ & $-0,10$ & 0,52 \\
Total de la escala & $-0,42$ & $-0,23$ & 0,49 & & & $\alpha=0,89$ & \\
(sin el ítem 15) & $-0,45$ & & & & \\
\hline
\end{tabular}

Nota. $\mathrm{A}=$ Asimetría $; \mathrm{C}=$ Curtosis; $r_{\mathrm{i} \text {-total }}=$ Correlación corregida entre la puntuación del ítem y el puntaje de la escala; $\alpha=$ Alfa de Cronbach. 
Tabla 2

Análisis Factorial Exploratorio de la Escala de Actitudes hacia la Educación Multicultural

\begin{tabular}{|c|c|c|c|c|c|}
\hline Ítem & Factor 1 & Factor 2 & Factor 3 & Factor 4 & Factor 5 \\
\hline 14. Origina problemas de disciplina & 0,78 & & & & \\
\hline 8. Hace más difícil la tarea del profesor & 0,75 & & & & \\
\hline 22. Origina problemas de convivencia & 0,70 & & & 0,30 & \\
\hline 16. Dificulta la adaptación del profesor & 0,65 & & & & \\
\hline 6. Perjudica a las minorías & 0,58 & & & & \\
\hline 3. Provoca un mayor estrés en el profesor & 0,54 & & & & \\
\hline 5. Entorpece la programación de las materias & 0,44 & & 0,31 & & \\
\hline 10. Perjudica el nivel académico promedio & 0,44 & & 0,36 & & \\
\hline 12. Resulta mejor la educación monocultural & 0,30 & & & & \\
\hline 11. Los niños aceptan mejor las diferencias & & 0,81 & & & \\
\hline 24. Hace al profesor más tolerante & & 0,71 & & & \\
\hline 17. Aumenta el interés hacia otras culturas & & 0,65 & & & 0,30 \\
\hline 21. La escuela debe apoyar la diversidad & & 0,61 & & & \\
\hline 13. Favorece la innovación educativa & & 0,58 & 0,31 & 0,37 & \\
\hline 19. La escuela debe acoger la diversidad & & 0,49 & 0,65 & & \\
\hline 23. La escuela debe enseñar la cultura propia & & & 0,64 & & \\
\hline 20. Es mejor agrupar en clases separadas & 0,34 & 0,37 & 0,58 & & \\
\hline 18. Origina más problemas que ventajas & 0,48 & & 0,56 & & \\
\hline 2. Se trabajan contenidos más variados & & & & 0,82 & \\
\hline 7. Se utiliza mayor diversidad de materiales & & 0,46 & & 0,65 & \\
\hline 9. Origina mejores experiencias educativas & & 0,52 & 0,40 & 0,52 & \\
\hline 1. Favorece la socialización de los niños & & & & 0,41 & 0,73 \\
\hline 4. Los niños se adaptan mejor a los cambios & & 0,53 & & & 0,62 \\
\hline
\end{tabular}

Nota. Los pesos factoriales de los ítems que definen cada factor se destacan en negrita.

El quinto factor explica el $4,6 \%$ de la varianza y $\alpha$ es 0,66 . Incluye los ítems 1 y 4 y se ha denominado Ventajas sociales. Hace referencia a las consecuencias de la interacción entre niños de diferentes culturas.

Los índices de discriminación oscilan entre 0,38 y 0,69 , es decir, los ítems de cada uno de los factores presentan una adecuada capacidad de discriminación.

Del AFC resultó la solución completamente estandarizada que se muestra en la Tabla 3.

Para evaluar la bondad de ajuste del modelo se hicieron las siguientes consideraciones: a) no es aconsejable evaluar la bondad de ajuste de un modelo solamente a partir de un único índice de ajuste global (Bollen \& Long, 1993); b) los valores obtenidos para los distintos índices de ajuste fueron: $\chi^{2}$ $(239, N=117)=386,50, p=0,00$; RMSEA $=$
0,095; $\mathrm{CFI}=1,00 ; \mathrm{GFI}=0,97 ; \mathrm{AGFI}=0,96 ;$ c) el intervalo de confianza para el RMSEA fue de 0,068 a 0,106 ; d) los valores resultantes de los índices que tratan de evaluar la parsimonia del modelo y complementan la información aportada por el RMSEA (índice que se ve afectado por el número de parámetros) fueron para AIC: saturado $=600$; evaluado $=508,5$; independiente $=3079,76$; y para CAIC: saturado $=1574,5$; evaluado $=706,7$; independiente $=3157,7$. Se puede concluir que el modelo propuesto presenta un ajuste razonable, si se considera el conjunto de índices de bondad de ajuste recién expuestos y no únicamente alguno de ellos. Ello queda refrendado por el sentido teórico que muestran las relaciones presentadas en el modelo que, además, son todas significativamente distintas de cero $(p<0,05)$. Por esta razón, no se consideró necesario poner 
Tabla 3

Solución Completamente Estandarizada del Análisis Factorial Confirmatorio para la Escala de Actitudes hacia la Educación Multicultural

\begin{tabular}{|c|c|c|c|c|c|}
\hline \multirow{3}{*}{ Ítem } & \multicolumn{5}{|c|}{ Factor } \\
\hline & 1 & 2 & 3 & 4 & 5 \\
\hline & & & $\lambda_{\mathrm{x}}$ & & \\
\hline 14 & 0,80 & -- & -- & -- & -- \\
\hline 10 & 0,75 & -- & -- & -- & -- \\
\hline 8 & 0,74 & -- & -- & -- & -. \\
\hline 16 & 0,70 & -- & .- & .- & -. \\
\hline 5 & 0,60 & -- & -- & .- & -.- \\
\hline 22 & 0,59 & -- & -- & -- & 0,27 \\
\hline 3 & 0,55 & -- & -- & -- & -- \\
\hline 12 & 0,52 & -- & -- & -- & -- \\
\hline 6 & 0,42 & -- & -- & -- & -- \\
\hline 17 & -- & 0,88 & -- & -- & -- \\
\hline 21 & . - & 0,86 & -- & -- & -. \\
\hline 13 & .- & 0,83 & -. & .- & .- \\
\hline 24 & - & 0,74 & -- & -- & -. \\
\hline 11 & -- & 0,59 & -- & -- & -- \\
\hline 18 & -- & -- & 0,89 & -- & -- \\
\hline 20 & -- & -- & 0,71 & -- & -. \\
\hline 23 & -- & -- & 0,70 & -- & -. \\
\hline 19 & -- & 0,50 & 0,38 & .- & .- \\
\hline 9 & .- & .- & .- & 0,93 & .- \\
\hline 7 & -- & -- & -- & 0,84 & -- \\
\hline 2 & -- & -- & -- & 0,62 & -- \\
\hline 4 & -- & -- & -- & -- & 0,83 \\
\hline 1 & 0,17 & -. & -- & -- & 0,67 \\
\hline
\end{tabular}

Nota. Las $\lambda_{\mathrm{x}}$ de los ítems que definen cada factor se destacan en negrita.

a prueba otro modelo, dado que el objetivo era obtener evidencias de validez del modelo propuesto y no construir teoría a partir de criterios estadísticos de ajuste.

En síntesis, la escala está compuesta por 23 elementos que se agrupan en cinco factores. Comparando la estructura factorial que se muestra en la Tabla 2 con la solución completamente estandarizada de la Tabla 3 se observan algunas diferencias, pero la composición de los factores es semejante. La consistencia interna del instrumento es $\alpha=0,90$, oscilando según los factores entre 0,66 y 0,83 . El menor corresponde al Factor 5 y es aceptable porque únicamente lo componen dos elementos (Hair, Anderson, Tatham \& Black, 1998). Además, cada factor presenta suficiente capacidad discriminativa.
Análisis Descriptivos de las Actitudes hacia la Educación Multicultural

Por adición de las puntuaciones de los ítems que componen cada factor se generan las variables latentes multiculturalidad negativa, multiculturalidad positiva, papel de la escuela, efectos en el trabajo del aula y ventajas sociales. El análisis descriptivo de estas variables (ver Tabla 4) revela que los valores de asimetría y curtosis están comprendidos entre $-1,96$ y $+1,96$, salvo en la variable ventajas sociales. Es decir, la mayoría de las variables se ajusta razonablemente a la distribución normal.

En cada actitud evaluada, el tercio superior de las puntuaciones representa las actitudes positivas, el tercio inferior señala las actitudes negativas y las puntuacio- 
Tabla 4

Estadígrafos Descriptivos de las Variables de Actitudes hacia la Educación Multicultural

\begin{tabular}{lccccc}
\hline Variable & $M$ & $D T$ & $V$ & $A$ & $C$ \\
\hline Multiculturalidad negativa & 23,85 & 6,03 & 36,37 & $-0,19$ & $-0,03$ \\
Multiculturalidad positiva & 16,74 & 3,21 & 10,28 & $-1,07$ & 1,12 \\
Papel de la escuela & 13,03 & 2,63 & 6,94 & $-1,03$ & 0,77 \\
Efectos en el trabajo del aula & 10,26 & 1,93 & 3,73 & $-1,14$ & 0,82 \\
Ventajas sociales & 7,23 & 1,09 & 1,20 & $-1,68$ & 3,74 \\
\hline
\end{tabular}

Nota. $M=$ Media; $D T=$ Desviación típica; $V=$ Varianza; $A=$ Asimetría $C=$ Curtosis.

nes comprendidas entre ambos extremos, las actitudes intermedias. Por ejemplo, en multiculturalidad negativa, las puntuaciones entre 0 y 11 se categorizan como actitud negativa, las puntuaciones entre $12 \mathrm{y}$ 24 , como actitud intermedia y entre 25 y 36 , como actitud positiva.

En la Figura 1 se presenta un resumen del análisis de frecuencias. El 93,2\% de los individuos muestra puntuaciones en ventajas sociales que indican una tendencia positiva hacia la interacción entre niños de diferentes etnias, manifestando que favorece la socialización y que les prepara mejor para los cambios y el futuro. El $82,9 \%$ de los participantes puntúa en multiculturalidad positiva en el sentido de considerar que la educación multicultural hace a los niños más comprensivos con los grupos de personas "diferentes", que favorece la innovación educativa y que aumenta el interés de los alumnos por conocer otras culturas. En efectos en el trabajo del aula, el 82,1\% de los profesores en formación manifiesta una actitud positiva consistente en entender que en clases con niños de diferentes culturas se trabajan contenidos más variados $\mathrm{y}$ atractivos, que la diversidad de materiales utilizados resulta más motivadora para el aprendizaje y que se originan experiencias de aprendizaje más enriquecedoras. El 78,6\% de los participantes muestra una actitud positiva en papel de la escuela, es decir, están de acuerdo en que la escuela asuma la diversidad cultural y desestiman las ideas de que los niños de culturas minoritarias sean agrupados en clases separadas o que la escuela solo enseñe su propia cultura. El 47\% muestra una actitud positiva en multiculturalidad negativa, es decir, menos de la mitad de los participantes está en desacuerdo con los efectos negativos de la multiculturalidad en la labor del profesor y en el rendimiento académico de los alumnos. En esta variable, el 48,7\% de los estudiantes muestra actitudes intermedias, es decir, no se pronuncia a favor o en contra de la educación multicultural.

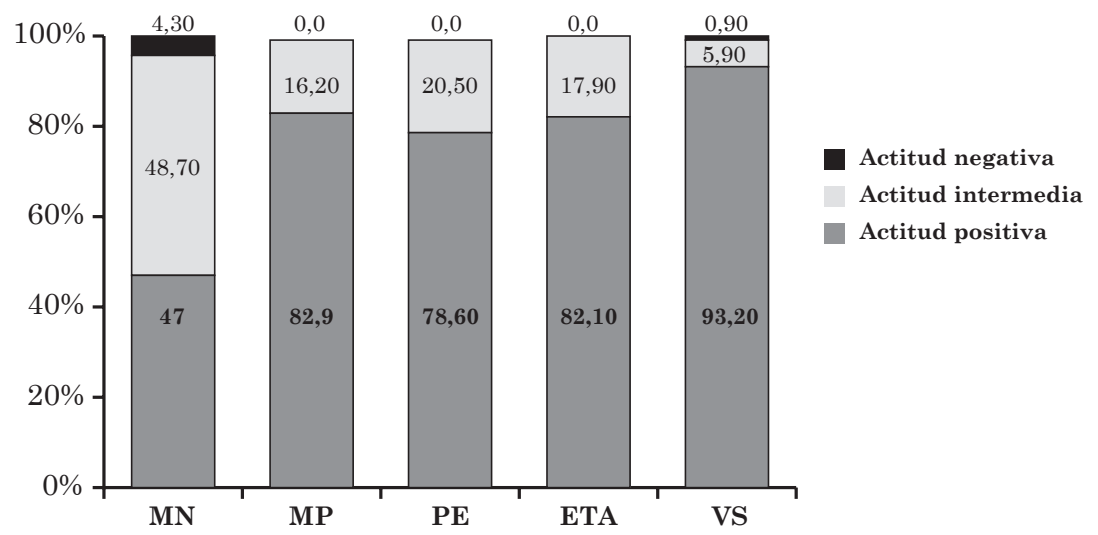

Figura 1. Distribución de frecuencias de las variables latentes.

Nota. $\mathrm{MN}=$ Multiculturalidad negativa, $\mathrm{MP}=$ Multiculturalidad positiva, $\mathrm{PE}=\mathrm{Papel}$ de la escuela, ETA = Efectos en el trabajo en el aula, VS = Ventajas sociales. 


\section{Análisis Diferenciales}

Los resultados de la prueba de Levene (ver Tabla 5) indican que no existen diferencias estadísticamente significativas por sexo en las varianzas de las variables analizadas, lo cual permite suponer el mismo grado de homogeneidad en ambos grupos.

Según se observa en la Tabla 6, los resultados indican que existen diferencias estadísticamente significativas por sexo en las variables multiculturalidad negativa $(t[115]=2,45, p=0,016)$ y papel de la escuela $(t[115]=2,34, p=0,021)$. Hay que tener en cuenta que cuanto más alto es el valor medio (M), más positiva es la actitud. Así, las mujeres desestiman en mayor medida que los hombres los efectos negativos de la multiculturalidad en la escuela. Además, las mujeres muestran una actitud más positiva que los hombres al considerar que la escuela debería asumir la diversidad cultural y al rechazar que los niños de culturas minoritarias estén en clases separadas o que la escuela solo enseñe su propia cultura.

En la Tabla 7 se presentan los resultados de los análisis diferenciales en función del tipo de relación mantenida con personas inmigrantes. En la misma se observa que existen diferencias significativas en todas las variables analizadas, pero dependiendo de los grupos que se comparen. En multiculturalidad negativa y en multiculturalidad positiva la media es mayor entre quie-

Tabla 5

Prueba de Levene para la Homogeneidad de las Varianzas de las Actitudes hacia la Educación Multicultural, según Sexo

\begin{tabular}{lcc}
\hline Variable & $F$ & $p$ \\
\hline Multiculturalidad negativa & 0,009 & 0,924 \\
Multiculturalidad positiva & 0,105 & 0,746 \\
Papel de la escuela & 0,816 & 0,368 \\
Efectos en el trabajo del aula & 0,158 & 0,692 \\
Ventajas sociales & 1,877 & 0,173 \\
\hline
\end{tabular}

Tabla 6

Prueba t de Student para el Contraste de Medias de las Actitudes hacia la Educación Multicultural según Sexo

\begin{tabular}{|c|c|c|c|c|c|c|c|}
\hline \multirow[b]{2}{*}{ Variable } & \multicolumn{4}{|c|}{ Descriptivos de Grupo } & \multicolumn{3}{|c|}{ Prueba $t$} \\
\hline & Sexo & $n$ & $M$ & $D T$ & $t$ & $g l$ & $p$ \\
\hline \multirow{2}{*}{ Multiculturalidad negativa } & Mujer & 77 & 24,82 & 5,91 & 2,45 & 116 & $0,016^{*}$ \\
\hline & Hombre & 40 & 22,00 & 5,89 & \multirow{2}{*}{1,37} & \multirow{2}{*}{115} & \multirow{2}{*}{0,174} \\
\hline \multirow{2}{*}{ Multiculturalidad positiva } & Mujer & 77 & 17,03 & 3,29 & & & \\
\hline & Hombre & 40 & 16,18 & 3,00 & \multirow{2}{*}{2,34} & \multirow{2}{*}{115} & \multirow{2}{*}{$0,021^{*}$} \\
\hline \multirow{2}{*}{ Papel de la escuela } & Mujer & 77 & 13,43 & 2,47 & & & \\
\hline & Hombre & 40 & 12,25 & 2,78 & \multirow{2}{*}{1,14} & \multirow{2}{*}{115} & \multirow{2}{*}{0,258} \\
\hline \multirow{2}{*}{ Efectos en el trabajo del aula } & Mujer & 77 & 10,40 & 1,96 & & & \\
\hline & Hombre & 40 & 9,98 & 1,86 & \multirow{2}{*}{0,93} & \multirow{2}{*}{115} & \multirow{2}{*}{0,354} \\
\hline \multirow{2}{*}{ Ventajas sociales } & Mujer & 77 & 7,30 & 1,08 & & & \\
\hline & Hombre & 40 & 7,10 & 1,13 & & & \\
\hline
\end{tabular}

$* p<0,05$ 
Tabla 7

Prueba U de Mann-Whitney para el Contraste de Medias de las Actitudes hacia la Educación Multicultural según el Tipo de Relación con Inmigrantes

\begin{tabular}{|c|c|c|c|c|c|}
\hline Variable & Comparación entre Tipos de Relación & $n$ & Rango promedio & $U$ & $p$ \\
\hline \multirow{4}{*}{$\mathrm{MN}$} & Ninguna / Barrio & $44 / 4$ & $23,45 / 36,00$ & 42,0 & 0,09 \\
\hline & Ninguna / Estudio o trabajo & $44 / 21$ & $28,59 / 42,24$ & 268,0 & $0,00 *$ \\
\hline & Ninguna / Actividades sociales & $44 / 19$ & $29,95 / 36,74$ & 328,0 & 0,17 \\
\hline & Ninguna / Personal & $44 / 29$ & $31,82 / 44,86$ & 410,0 & $0,01^{*}$ \\
\hline \multirow{4}{*}{ MP } & Ninguna / Barrio & $44 / 4$ & $23,48 / 35,75$ & 43,0 & 0,09 \\
\hline & Ninguna / Estudio o trabajo & $44 / 21$ & $28,48 / 42,48$ & 263,0 & $0,00^{*}$ \\
\hline & Ninguna / Actividades sociales & $44 / 19$ & $31,13 / 34,03$ & 379,5 & 0,56 \\
\hline & Ninguna / Personal & $44 / 29$ & $31,10 / 45,95$ & 378,5 & $0,00 *$ \\
\hline \multirow{4}{*}{$\mathrm{PE}$} & Ninguna / Barrio & $44 / 4$ & $23,45 / 36,00$ & 42,0 & 0,09 \\
\hline & Ninguna / Estudio o trabajo & $44 / 21$ & $26,69 / 46,21$ & 184,5 & $0,00 *$ \\
\hline & Ninguna / Actividades sociales & $44 / 19$ & $27,49 / 42,45$ & 219,5 & $0,00^{*}$ \\
\hline & Ninguna / Personal & $44 / 29$ & 30,92 / 46,22 & 370,5 & $0,02^{*}$ \\
\hline \multirow{4}{*}{ ETA } & Ninguna / Barrio & $44 / 4$ & $23,83 / 31,88$ & 58,5 & 0,28 \\
\hline & Ninguna / Estudio o trabajo & $44 / 21$ & $30,19 / 38,88$ & 338,5 & 0,07 \\
\hline & Ninguna / Actividades sociales & $44 / 19$ & $29,43 / 37,95$ & 305,0 & 0,08 \\
\hline & Ninguna / Personal & $44 / 29$ & $32,42 / 43,95$ & 436,5 & $0,02^{*}$ \\
\hline \multirow{4}{*}{ VS } & Ninguna / Barrio & $44 / 4$ & $24,07 / 29,25$ & 69,0 & 0,50 \\
\hline & Ninguna / Estudio o trabajo & $44 / 21$ & $30,97 / 37,26$ & 372,5 & 0,17 \\
\hline & Ninguna / Actividades sociales & $44 / 19$ & $29,18 / 38,53$ & 294,0 & $0,03^{*}$ \\
\hline & Ninguna / Personal & $44 / 29$ & $34,35 / 41,02$ & 521,5 & 0,15 \\
\hline
\end{tabular}

Nota. MN = Multiculturalidad negativa; $\mathrm{MP}=$ Multiculturalidad positiva; $\mathrm{PE}=$ Papel de la escuela; ETA = Efectos en el trabajo del aula; VS = Ventajas sociales.

$* p<0,05$.

nes estudian o trabajan con inmigrantes o quienes mantienen relaciones personales con ellos, respecto de los que no tienen relación alguna. En papel de la escuela, además de las anteriores, se observan diferencias entre quienes no tienen relación con inmigrantes y quienes tienen relaciones de tipo social, siendo la media más elevada en estos últimos. En efectos en el trabajo del aula solo hay una diferencia significativa entre el grupo sin relación con el grupo de relación personal, a favor de estos últimos. Y en ventajas sociales las diferencias estadísticamente significativas se observan entre los grupos sin relación y con relaciones sociales. En consecuencia, se deduce que los individuos que se han relacionado con inmigrantes manifiestan una mejor actitud hacia la educación multicultural, en comparación con los que no se han relacionado con ellos.

\section{Discusión}

La Escala de Actitudes hacia la Educación Multicultural (Cabrera et al., 1997a), aplicada a estudiantes universitarios chilenos, presenta índices de consistencia interna y discriminación adecuados, aunque su estructura factorial difiere de la aportada por los autores originales. Esto es razonable considerando que los participantes de este estudio pertenecen a un contexto distinto, en donde la inmigración, las variables sociales -entendidas como las normas y leyes adoptadas por una determinada sociedad- y las relaciones históricas entre los diversos grupos (Molero, Cuadrado \& Navas, 2003; Rodríguez, Betancor \& Delgado, 2009) presentan características propias. Indagar acerca de cómo operan las variables sociales constituye una línea de trabajo de cara 
al futuro. También, las diferencias pueden deberse a que Cabrera et al. (1997b) plantearon las diferentes actitudes desde un análisis teórico y no, como en este estudio, a través de análisis empíricos o a que en la investigación original los participantes eran profesores en ejercicio, con más de tres años de experiencia y más de la mitad, con experiencia en multiculturalidad, y no profesores en formación.

Hay que destacar que si bien los análisis realizados sugieren que la Escala de Actitudes hacia la Educación Multicultural (Cabrera et al., 1997a) es un instrumento confiable para abordar el estudio de las actitudes en el contexto chileno, sería conveniente estudiar el comportamiento del instrumento en una muestra más amplia de la población.

Aunque los resultados indican bajos niveles de prejuicio manifiesto, se debe considerar que alrededor del $20 \%$ de los profesores en formación manifestaron actitudes intermedias. Quizás esto podría reflejar una forma enmascarada de prejuicio o de hostilidad sutil hacia el exogrupo (Dovidio \& Gaertner, 1991, 1998; García, Navas, Cuadrado \& Molero, 2003; Navas, 1998; Pettigrew \& Meertens, 1995; Rueda, Navas \& Gómez, 1995) que sería digno de analizar, considerando que se trata de futuros maestros, que no solo deben trasmitir y desarrollar nuevas capacidades sino, también, deben respetar la cultura de sus alumnos y estimularlos a respetar las diversas formas culturales que existen. Se podría considerar también la influencia de las percepciones acerca de lo que se considera socialmente deseable, que podría afectar a los participantes. Según Morales (2003), la deseabilidad social puede enmascarar actitudes xenófobas latentes. O, quizás, se podría explicar en función de la indecisión de los individuos o de su falta de opinión formada al respecto.

No obstante, es necesario precisar que estos hallazgos no pueden ser generalizados a todos los estudiantes de pedagogía chilenos, ya que la técnica de muestreo empleada no permite generalizar los resultados y porque la manifestación del prejuicio se caracteriza por variar entre contextos.
Las diferencias encontradas entre hombres y mujeres en multiculturalidad negativa y papel de la escuela podrían explicarse por variables psicológicas (características de personalidad), por variables sociales (la calidad de las relaciones mantenidas con personas de grupos culturales distintos al propio) o por variables cognitivas (el tipo de información que se tiene sobre la inmigración y la interpretación que se realiza de esta), como ha sido indicado por Batson et al. (1997), Galinsky y Moskowitz (2000) o McFarland (2001, Febrero). El motivo por el que las mujeres presentan actitudes más positivas que los hombres puede constituir una línea de trabajo en estudios futuros.

Las diferencias que se observan en todas las variables objeto de estudio que favorecen a quienes se han relacionado con inmigrantes concuerdan con la hipótesis que plantea que un mayor contacto con el exogrupo favorece el desarrollo de actitudes más positivas hacia sus miembros (Allport, 1954; Kalin \& Berry, 1982; Pettigrew \& Tropp, 2000). El tipo de relación no introduce diferencias del mismo modo. Los resultados indican que relaciones más íntimas, con un mayor conocimiento mutuo, se asocian a actitudes más positivas ante la multiculturalidad en la escuela.

Aunque los resultados obtenidos aportan información valiosa sobre la actitud de los profesores en formación, estas actitudes merecen ser analizadas con mayor profundidad en futuros estudios y en una muestra mayor que abarque a todas las regiones del país.

Igualmente sería interesante analizar si la ideología política de los participantes, si su visión del mundo (global o local) o si la percepción subjetiva de bonanza o estabilidad económica del país introduce diferencias en sus actitudes hacia la multiculturalidad, tal como ocurre en otros contextos (Haubert \& Fussell, 2006; Ward \& Masgoret, 2008; Wilkes, Guppy \& Farris, 2008). También es importante diferenciar las actitudes dirigidas a distintos grupos culturales (peruanos, argentinos, colombianos, etc.), ya que estos podrían evocar diversas emociones y distintas conductas (Cottrell \& Neuberg, 2005; Neuberg \& Cottrell, 2002). Estas son cuestiones a abordar en futuras investigaciones. 
Para finalizar, como implicación práctica derivada de los resultados, cabe plantearse la posibilidad de llevar a cabo un programa para el cambio de actitudes en los futuros profesores. Esto se justificaría porque se han detectado actitudes intermedias en los participantes que podrían indicar la existencia de prejuicio encubierto.

En la etapa de formación inicial universitaria se pueden trabajar mejor las actitudes y, sobre todo, se pueden desarrollar programas de sensibilización que permitan al futuro docente acercarse al tema de la inmigración desde una perspectiva formativa y evitar, así, que los profesores, inconscientemente, reproduzcan en sus clases la marginación de las minorías (Gagliardi, 1995).

\section{Referencias}

Allport, G. W. (1954). The nature of prejudice. Reading, MA: Addison-Wesley.

Archer, P. (1981). The influence of pupil's social class on teacher rating of reading attainment. Resources in Education, 16, ED 195973.

Aronson, J., Fried, C. B. \& Good, C. (2002). Reducing the effects of stereotype threat on African American college students: The role of theories of intelligence. Journal of Experimental Social Psychology, 38, 113-125.

Aronson, J., Lustina, M. J., Good, C., Keough, K., Steele, C. M. \& Brown, J. (1999). When white men can't do math: Necessary and sufficient factors in stereotype threat. Journal of Experimental Social Psychology, 35, 29-46.

Barron, I. (2007). An exploration of young children's ethnic identities as communities of practice. British Journal of Sociology of Education, 28, 739-752.

Batson, C. D., Polycarpou, M. P., Harmon-Jones, E., Imhoff, H. J., Mitchener, E. C., Bednar, L. L. et al. (1997). Empathy and attitudes: Can feeling for a member of a stigmatized group improve feelings toward the group? Journal of Personality and Social Psychology, 72, 105-118.

Bollen, K. A. \& Long, J. S. (1993). Testing structural equation models. London: Sage Publications.

Brown, R., Eller, A., Leeds, S. \& Stace, K. (2007). Intergroup contact and intergroup attitudes: A longitudinal study. European Journal of Social Psychology, 37, 692-703.

Brown, R. \& Hewstone, M. (2005). An integrative theory of intergroup contact. Advances in Experimental Social Psychology, 37, 255-343.

Brunsma, D. L. (2006). Public categories, private identities: Exploring regional differences in the biracial experience. Social Science Research, 35, 555-575.

Cabrera, F., Espín, J., Marín, M. \& Rodríguez, M. (1997a). La escala de actitudes para el profesorado. En M. Bartolomé (Coord.), Diagnóstico a la escuela multicultural (pp. 343-348). Barcelona: Cedecs.
Cabrera, F., Espín, J., Marín, M. \& Rodríguez, M. (1997b). Actitudes del profesorado ante la educación multicultural. En M. Bartolomé (Coord.), Diagnóstico a la escuela multicultural (pp. 181-187). Barcelona: Cedecs.

Cottrell, C. A. \& Neuberg, S. L. (2005). Different emotional reactions to different groups: A sociofunctional threat-based approach to "prejudice". Journal of Personality and Social Psychology, 88, 770-789.

Chile, Instituto Nacional de Estadísticas (2003). Características demográficas. En Chile, Instituto Nacional de Estadísticas, Censo 2002. Síntesis de resultados (pp. 9-19). Santiago, Chile: Autor. Extraído el 30 Enero, 2009, de http://www.ine.cl/ cd2002/sintesiscensal.pdf

Chile, Presidencia de la República (2008). Política Nacional Migratoria. Santiago, Chile: Autor. Extraído el 1 Febrero, 2009, de http://www. interior.gov.cl/filesapp/Politica_Nacional_ Migratoria.pdf

Díaz, A. (2002). Interacción y multiculturalidad en la escuela. En M. P. Pardo de León \& L. Méndez (Eds.), Psicología de la educación multicultural (pp. 279-309). Madrid: Universidad Nacional de Educación a Distancia.

Díaz, M., Baraja, A. \& Royo, P. (1996). Estudio sobre la integración escolar de alumnos extranjeros y el aprendizaje del castellano como segunda lengua. En M. Díaz (Coord.), Escuela y tolerancia (pp. 101-154). Madrid: Pirámide.

Dovidio, J. F. \& Gaertner, S. L. (1991). Changes in the expression and assessment of racial prejudice. En H. J. Knopke, R. J. Norrell \& R. W. Rogers (Eds.), Opening doors: Perspectives on race relations in contemporary America (pp. 119-448). Tuscaloosa, AL: The University of Alabama Press.

Dovidio, J. F. \& Gaertner, S. L. (1998). On the nature of contemporary prejudice: The causes, consequences, and challenges of aversive racism. En J. L. Eberhardt \& S. T. Fiske (Eds.), Confronting racism: The problem and the response (pp. 3-32). London: Sage Publications.

Fernández, E., Aguilar, M. M. \& Gutiérrez, M. L. (2002). Educación multicultural: dimensiones actitudinales. Revista Electrónica Interuniversitaria de Formación del Profesorado, 5(1), Artículo 14. Extraído el 1 Febrero, 2009, de http://www.aufop. com/aufop/uploaded_files/articulos/1227717396. pdf

Gagliardi, R. (1995). La educación multicultural y la formación de maestros. Genève: Organización de las Naciones Unidas para la Educación, la Ciencia y la Cultura, Oficina Internacional de Educación. Extraído el 13 Junio, 2008, de http://www. ibe.unesco.org/fileadmin/user_upload/archive/ publications/teachers/report_31.pdf

Galinsky, A. D. \& Moskowitz, G. B. (2000). Perspectivetaking: Decreasing stereotype expression, stereotype accessibility, and in-group favoritism. Journal of Personality and Social Psychology, 78, 708-724.

Gallimore, R. \& Goldenberg, C. (2001). Analyzing cultural models and settings to connect minority achievement and school improvement research. Educational Psychologist, 36, 45-56. 
García, M. C., Navas, M. S., Cuadrado, I. \& Molero, F. (2003). Inmigración y prejuicio: actitudes de una muestra de adolescentes almerienses. Acción Psicológica, 2, 137-147.

Glick, J. E. \& Hohmann-Marriott, B. (2007). Academic performance of young children in immigrant families: The significance of race, ethnicity, and national origins. International Migration Review, 41, 371-402.

Glick, J. E. \& White, M. J. (2003). The academic trajectories of immigrant youths. Analysis within and across cohorts. Demography, 40, 749-784.

Hair, J. F., Anderson, R. E., Tatham, R. L. \& Black, W. C. (1998). Multivariate data analysis ( $5^{\text {th }}$ ed.). Upper Saddle River, NJ: Prentice Hall.

Haubert, J. \& Fussell, E. (2006). Explaining proimmigrant sentiment in the U. S.: Social class, cosmopolitanism, and perceptions of immigrants. International Migration Review, 40, 489-507.

Jackson, P. W. (1968). Life in classroom. New York: Holt, Rinehart and Winston.

Jordán, J. A. (1994). La escuela multicultural. Barcelona: Paidós Ibérica.

Jöreskog, K. G. (2001). Analysis of ordinal variables 2 : Cross-sectional data. Texto presentado en el Taller Structural Equation Modeling with LISREL 8.51, Friedrich-Schiller-Universität Jena, Alemania.

Kalin, R. \& Berry, J. W. (1982). The social ecology of ethnic attitudes in Canada. Canadian Journal of Behavioural Science, 14, 97-109.

León del Barco, B., Mira, A. R. \& Gómez, T. (2007). Evaluación de las opiniones sobre la inmigración y la multiculturalidad en la escuela de alumnos de magisterio. Revista Electrónica de Investigación Psicoeducativa, 5, 259-282.

McFarland, S. (2001, Febrero). Prejudiced people: Individual differences in explicit prejudice. Ponencia presentada en la Annual Meeting of the Society for Personality and Social Psychology, San Antonio, TX, Estados Unidos.

Molero, F., Cuadrado, I. \& Navas, M. S. (2003). Las nuevas expresiones del prejuicio racial: aspectos teóricos y empíricos. En J. F. Morales \& C. Huici (Coords.), Estudios de psicología social (pp. 83117). Madrid: Universidad Nacional de Educación a Distancia.

Molero, F., Navas, M. \& Morales, J. F. (2001). Inmigración, prejuicio y exclusión social: reflexiones en torno a algunos datos de la realidad española. International Journal of Psychology and Psychological Therapy, 1, 11-32.

Morales, J. F. (2003). El estudio del prejuicio en psicología social. En J. F. Morales \& C. Huici (Coords.), Psicología social (pp. 219-234). Madrid: McGraw-Hill.

Navas, L., Sampascual, G. \& Castejón, J. L. (1991). Las expectativas de profesores y alumnos como predictores del rendimiento académico. Revista de Psicología General y Aplicada, 44, 231-239.

Navas, M. S. (1998). Nuevos instrumentos de medida para el nuevo racismo. Revista de Psicología Social, 13, 233-239.
Neuberg, S. L \& Cottrell, C. A. (2002). Intergroup emotions: A sociofunctional approach. En D. M. Mackie \& E. R. Smith (Eds.), From prejudice to intergroup emotions: Differentiated reactions to social groups (pp. 265-283). New York: Psychology Press.

Pantoja, A. (2006). Against the tide? Core American values and attitudes toward US immigration policy in the mid-1990s. Journal of Ethnic and Migration Studies, 32, 515-531.

Pettigrew, T. F. \& Meertens, R. W. (1995). Subtle and blatant prejudice in Western Europe. European Journal of Social Psychology, 25, 57-75.

Pettigrew, T. F. \& Tropp, L. R. (2000). Does intergroup contact reduce prejudice? Recent meta-analytic findings. En S. Oskamp (Ed.), Reducing prejudice and discrimination (pp. 93-114). Mahwah, NJ: Lawrence Erlbaum Associates.

Quesille, A. (2007). Inmigrantes, refugiados y derechos humanos. En N. Espejo (Coord.), Informe anual sobre derechos humanos en Chile. Hechos 2006 (pp. 317-362). Santiago, Chile: Universidad Diego Portales.

Ridao, R. (2006). Inmigración y educación. A propósito de su representación discursiva. En M. Lario (Coord.), Medios de comunicación e inmigración (pp. 215-235). Murcia, España: Convivir Sin Racismo.

Rodríguez, A., Betancor, V. \& Delgado, N. (2009). La norma social sobre la expresión de prejuicio explícito hacia diferentes grupos sociales. Revista de Psicología Social, 24, 17-27.

Roth, W. D. (2005). The end of the one drop rule? Labeling of multiracial children in black intermarriages. Sociological Forum, 20, 35-67.

Rueda, J. F., Navas, M. S. \& Gómez, C. (1995). Las nuevas expresiones del racismo: adaptación de una escala de prejuicio sutil. En J. C. Sánchez \& A. M. Ullán (Comps.), Procesos psicosociales básicos y grupales (pp. 357-370). Salamanca, España: Eudema.

Steele, C. M. (1997). A threat in the air: How stereotypes shape intellectual identity and performance. American Psychologist, 52, 613-629.

Van Matre, J. C., Valentine, J. C. \& Cooper, H. (2000). Effect of students' after-school activities on teachers' academic expectancies. Contemporary Educational Psychology, 25, 167-183.

Ward, C. \& Masgoret, A-M. (2008). Attitudes toward immigrants, immigration, and multiculturalism in New Zealand: A social psychological analysis. International Migration Review, 42, 227-248.

Wilkes, R., Guppy, N. \& Farris, L. (2008). "No thanks, we're full": Individual characteristics, national context, and changing attitudes toward immigration. International Migration Review, 42, 302-329.

Woolfolk, A. (2006). Psicología educativa (5 ${ }^{\mathrm{a}}$ ed.). Ciudad de México: Pearson Educación.

Fecha de recepción: Marzo de 2009.

Fecha de aceptación: Diciembre de 2009. 